\title{
Practice of Malaria Prevention and Control Methods and Associated Factors Among Rural Households in West Belessa District 2019
}

Tsigie Baye Aragie ( $\nabla$ deacontsige@gmail.com )

University of Gondar

Haileab Fekadu wolde

University of Gondar

\section{Research}

Keywords: Malaria, practice, and prevention

Posted Date: January 28th, 2021

DOl: https://doi.org/10.21203/rs.3.rs-153846/v1

License: (c) (i) This work is licensed under a Creative Commons Attribution 4.0 International License.

Read Full License 


\section{Abstract}

Background: In Ethiopia, the burden of malaria continues to cause a substantial number of morbidity and mortality. Communities' practices of malaria prevention and control methods contribute immensely to sustainable control of malaria. This evidence is quite limited in the study area. Hence, this study aimed to assess Practice of malaria prevention and control methods and associated factors among rural household in west belessa district, northwest Ethiopia, 2019.

Method: Community-based cross-sectional mixed quantitative and qualitative study was conducted from April to June 2019, in West Belessa district, North West Ethiopia. Multistage sampling was used to select a 740 sample size. A structured questionnaire was used for the quantitative component and a semistructured questionnaire for the qualitative component. Quantitative data collected by interviewing and qualitative data using focus group discussion. Quantitative data was coded and entered using Epi info software and analyzed using SPSS. The binary logistic regression model was fitted to identify the associated factors. Odds Ratio with 95\% Confidence Interval was used to assess the strength of association. The qualitative data was transcribed manually using the thematic approach.

Result: A total 738 subjects included with $99.7 \%$ response rate. $50.9 \%$ of respondents had good practice of malaria prevention and control methods. LLINs and IRS were practiced by $21.1 \%$ and $80.5 \%$ respectively. Poorest wealth quintiles [AOR $=0.45,95 \% \mathrm{Cl}: 0.27,0.76]$, poor wealth quintiles [AOR $=0.51$, $95 \% \mathrm{Cl}: 0.30,0.88$ ], medium wealth quintiles [AOR $=0.24,95 \% \mathrm{Cl}: 0.14,0.42]$ and wealthy wealth quintile [AOR $=0.21,95 \% \mathrm{Cl}: 0.12,0.36]$, living in Menti Kebele [AOR $=3.88,95 \% \mathrm{Cl}: 2.43,6.20]$, female sex [AOR = $0.65,95 \% \mathrm{Cl}: 0.47,0.90$ ], illiterate educational status [AOR $=0.34,95 \% \mathrm{Cl} 0.16,0.72$ ] knowledge level poor [AOR $=0.52,95 \% \mathrm{Cl}: 0.36,0.75]$ were significantly associated with good malaria prevention practice. All of the FGD participants participated at least one malaria prevention method.

Conclusion: There were good IRS and environmental management malaria prevention practices, however, LLINs and other malaria prevention methods were poorly practiced. Wealth index, respondents living kebele, female sex, educational status, and respondents' malaria prevention knowledge are predictors for the practice of malaria prevention.

\section{Background}

Malaria is a protozoan disease transmitted by the bite of infected Anopheles mosquitoes.(1) Globally, there were an estimated 229 million new malaria cases and about 409,000 malaria-related deaths in 2019.(2) Malaria is one of the leading causes of morbidity and mortality in Africa. The world health organization (WHO) African Region bearing the highest burden of malaria cases (94\%) and deaths (94\%). (2)

In Ethiopia, the burden of malaria continues to cause a substantial number of morbidity and mortality which accounts for most outpatient visits, and it has been one of the main causes of hospitalization and deaths in the country. $(3,4)$ Approximately 60 percent of the Ethiopian population lives in malarious areas, 
and 68 percent of the country's landmass is favorable for malaria transmission.(5) Malaria prevention practices of communities contribute immensely to sustainable control of endemic diseases such as malaria. Strategic malaria control involves primary prevention which focuses on vector control specifically through the consistent use of insecticide-treated nets and willingness to make the indoor residual spray.(6)

Ethiopia had planned to eliminate malaria in the country by 2030.(7) To achieve this goal the first option is vector monitoring and controlling through Entomological monitoring.(7) The first target of the Ethiopian Ministry of Health is $100 \%$ household coverage of at least one LLINs per two family members in the household in all malarious areas.(8) Similarly, FMOH aims to provide $100 \%$ IRS coverage as a key malaria prevention measure in areas where the malaria burden is high.(7) According to the new FMOH malaria risk stratification, $14.8 \%$ of the country's total population is targeted for IRS.(8) Other vector control activities including larval control through environmental management and chemical larvicides are also practiced in areas where such interventions are appropriate and expected to have a significant impact.(8) However in 2014 only overall, $32 \%$ of households in the malarious area have reached universal LLINs coverage, and $64 \%$ of households had at least one LLINs.(8)

Evidence from different studies has shown that the practice of malaria prevention and control methods were affected by different factors such as, employment status, marital status, religion, level of education, gender, age, wealth index, the structure of respondents' houses, and LLINs received by the household.(912)

Evidence about practice of malaria prevention and control methods among households in rural malarious areas of west Belessa district is quite limited. Hence, the present study aimed to assess the Practice of malaria prevention and control methods and associated factors among rural households in west belessa district, northwest Ethiopia, 2019. This study would help to evaluate the current malaria control programs in the study setup. If properly utilized, this information will urge the decision-makers to strengthen malaria control interventions effectively and efficiently.

\section{Methods}

\section{Study design and Setting}

A community-based cross-sectional mixed quantitative study and qualitative study was conducted from April to June 2019. This study was conducted in West Belessa district which is found in Amhara Regional State, North West Ethiopia. Ethiopia is one of the countries in East Africa with 1.104 million $\mathrm{km}^{2}$ areas and $112,078,730$ population in 2019. It has 9 regional states and 670 rural districts. West Belessa is one of these districts.

West Belessa is, situated at an altitude of 1501 to 3000 meters above sea level. The capital city of West Belessa is Arbaya. It is located at a latitude and longitude of $12^{\circ} 15^{\prime} \mathrm{N}$ and $37^{\circ} 45^{\prime} \mathrm{E}$ respectively. High 
Rainfall is registered from June to August with a shortage and heavy rain intermittently. The mean monthly temperature is $33.5^{\circ} \mathrm{c}$. There are mountainous, lowlands, and water bodies including a known river Mena in the district. The district has 3 climatic zones, which are hot zones (kola) $60 \%$, cold zones (dega) 35\%, and moderate zones (weyna dega) 5\%. From the total district land area, $92.9 \%$ are malarious. In 2019 the estimated total population of the district was 197,326. (Figure)

\section{Study population and sampling technique}

The source population for this study was all households in 23 malarious rural kebeles. There are a total of 25 kebeles in the district. The study population was all households in 5 selected malarious kebeles. The sample size for the survey was determined using a single population formula using a study done in Areka, Ethiopia which had $67.7 \%$ good practice.(13) $95 \% \mathrm{Cl}$, and a margin of error of 0.05 then 336 has been calculated. By adding a 10\% non-response rate and design effect 2 the final sample size became 740. There are 25 total kebeles in West Belessa. First of all these kebeles were divided into 3 clusters (Malarious, non-malarious and urban). From the rural malarious kebeles' 5 kebeles were selected by using the lottery method. The sample size was allocated proportionally based on Keble's population. Using household registration from health posts, we selected households by systematic sampling every 15 households. The interviewees are household heads.

\section{Inclusion and Exclusion criteria}

Inclusion criteria: All households' head in the selected kebeles and have been living there for at least 6 months before the interview.

Exclusion criteria: Very sick individuals who were unable to communicate excluded from the study Data Collection Methods and data quality control

The data collection tool was adopted from 2004 WHO/UNICEF guidelines for core population coverage survey with some modifications.(14) Originally prepared in English then translated into the local language (Amharic) then back to English to ensure reliable information. Twelve numerators who had graduated from college were recruited and trained about the purpose of the study; how to approach respondents; how to obtain written consent and an overview of malaria prevention methods for one day. An interviewer-administered questionnaire was used. Completeness of the questionnaire was checked every day and incomplete questionnaires were returned to the data collectors on the following day for correction by re-visiting the households. Absence households were revisited on the following days. Pretesting was conducted in one of the malarious kebeles out of the study area.

A total of 4 FGDs were conducted in 4 kebeles (Abay tera, Kalay, Aswagari, and Menti kebeles).

Participants with full of information about malaria prevention and control methods were selected for the discussion by using health extension workers as a key informant in each kebele. The FGD had 6 to 10 participants in each group and comprised a total of 30 study participants in 4 groups. Each FGD was 
conducted by recording sounds and taking notes. The selected interviewees were expected to answers the semi-structured questionnaires. The content of semi-structured questionnaires were issues about knowledge of malaria prevention and control methods.

\section{Variables of the study}

Practice of malaria prevention and control was a dependent variable of the study.

Practice: A study participant who scored above or equal to the median score of Practice questions was considered as having good Practice and others considered as having poor Practice.(13) The media was 7 out of 11 malaria prevention practice questions.

Independent variables: Age; sex, marital status, occupation, religion, ethnicity, address, family size, educational status, and wealth index.

Wealth quintiles: determined by using durable household assets. A total of 18 different durable assets were identified and assigned as dummy variables. After adjusting and coding we used multivariate analysis i.e. principal component analysis after we re-categorized into five different wealth quintiles, each with an approximately equal number of households.

\section{Data processing and Analysis}

Quantitative data entered and coded using Epi info version 7 and exported to SPSS version 20. Variables were analyzed using logistic regression in SPSS to determine the association between the variables determined by looking at the level of significance of 0.05 with a $95 \%$ Confidence Interval $(\mathrm{Cl})$.

Qualitative data were transcribed in the original language of the interview first word by word from the audiotapes and field notes, then it was translated to English for analysis. The primary theme was produced through manual coding using a pen of different colors, then it was pooled into broader concepts to form main themes.

\section{Ethical Consideration}

Approval to carry out the study was sought and obtained from the University of Gondar Ethics Review Board. Written consent was obtained from all study participants after a detailed explanation of the purpose of the study.

\section{Result}

\section{Socio-demographic characteristics}

A total of 738 households have participated in this study with a response rate of $99.7 \%$. Out of the total participants, 410 (55.6\%) were male. The mean age of the respondents was 40.2 (SD $= \pm 12.24$ ) years. Almost all of respondents $733(99.3 \%)$ were orthodox Christian, and the majority of the respondents $664(90.0 \%)$ were married. Concerning the educational status majority, $648(87.8 \%)$ of the participants 
were illiterate. Above half of the respondents, 438 (58.9\%) had 4-6 family members in their house. The mean household member was 5.01 (SD \pm 1.72 ). The economic status of the respondents was assessed using the wealth index which showed, $19.0 \%, 20.3 \%, 19.2 \%, 18.8 \%$, and $22.6 \%$ of the respondents ranked as wealthiest, wealthy, medium, poor, and poorest respectively. (Table 1) 
Table 1

SOCIO DEMOGRAPHIC CHARACTERISTICS OF RESPONDENTS FOR KNOWLEDGE AND PRACTICE TOWARDS MALARIA PREVENTION IN WEST BELESA, AMHARA REGION, ETHIOPIA, 2019.

\begin{tabular}{|c|c|c|c|}
\hline Variable & $N=766$ & Frequency & percent \\
\hline \multirow[t]{6}{*}{ Age } & $18-24$ & 49 & 6.6 \\
\hline & $25-34$ & 193 & 26.2 \\
\hline & $35-44$ & 234 & 31.7 \\
\hline & $45-54$ & 165 & 22.4 \\
\hline & $55-64$ & 66 & 8.9 \\
\hline & $>64$ & 31 & 4.2 \\
\hline \multirow[t]{2}{*}{ Sex } & Male & 410 & 55.6 \\
\hline & Female & 328 & 44.4 \\
\hline \multirow[t]{2}{*}{ Religion } & Orthodox & 733 & 99.3 \\
\hline & Others & 5 & 0.7 \\
\hline \multirow[t]{3}{*}{ Educational status } & Illiterate & 648 & 87.8 \\
\hline & Literate & 48 & 6.5 \\
\hline & Formal education & 42 & 5.7 \\
\hline \multirow[t]{4}{*}{ Marital status } & Married & 664 & 90.0 \\
\hline & Divorced & 26 & 3.5 \\
\hline & Not married & 35 & 4.7 \\
\hline & Widowed & 13 & 1.8 \\
\hline \multirow[t]{3}{*}{ Number of family members } & $<4$ & 157 & 21.3 \\
\hline & $4-6$ & 435 & 58.9 \\
\hline & $>6$ & 146 & 19.8 \\
\hline \multirow[t]{5}{*}{ Wealth index } & Poorest & 167 & 22.6 \\
\hline & Poor & 139 & 18.8 \\
\hline & Medium & 142 & 19.2 \\
\hline & Wealthy & 150 & 20.3 \\
\hline & Wealthiest & 140 & 19.0 \\
\hline
\end{tabular}




\section{Respondents Practice on Malaria prevention and control methods.}

Most of the households $582(78.9 \%)$ did not have LLINs. A total of 212 LLINs were identified in $156(21.1 \%)$ households; of which $100(64.1 \%)$ received one LLINs and the remaining $56(35.9 \%)$ households received two LLINs from the government. Regarding utilization out of the mosquito bed net owners, $107(68.6 \%$ ) of them used the mosquito bed net in the previous night of data collection. Of the total respondents, $22(3 \%)$ of them used LLINs for other purposes. (Table 2)

In this survey the most frequently used malaria prevention method was IRS (80.5\%) of which $130(17.8 \%)$ were re-plastered within six months after spray operation has been carried out. and the least frequently used malaria prevention methods were Closing windows and doors early in the evening (3.8\%). In this study, $77.0 \%$ of the respondents participated in environmental measures such as compact and drainage to control malaria and $40.2 \%$ of the respondents participated in clearing the vegetation. Above half $408(55.3 \%)$ of the respondents have been participated in epidemic control. (Table 2 )

Overall total respondents 379 (50.9\%) of respondents had good practices (with 95\% $\mathrm{Cl}, 47.2-54.5$ ) and $362(49.1 \%)$ of the respondents had poor practice towards malaria prevention and control overall. The median practices score for all respondents was 4 out of a possible 11 points $(\mathrm{IQR}=2-4)$

Table 2

practice on malaria prevention in west Belesa, North West Region, Ethiopia, 2019.

\begin{tabular}{|lcc|}
\hline Practices toward malaria prevention and Control methods & N=738 & Percent \\
\hline At least one LLIN & 156 & 21.1 \\
\hline Use of LLIN in the previous night & 107 & 14.5 \\
\hline IRS & 594 & 80.5 \\
\hline Use of mosquito proofing in windows and doors & 156 & 21.1 \\
\hline Closing windows and doors early at night & 28 & 3.8 \\
\hline Environmental management (compact and drainage) to prevent malaria & 568 & 77.0 \\
\hline Clear the vegetation & 297 & 40.2 \\
\hline Participated in Epidemic control & 408 & 55.3 \\
\hline Re-plastering the wall in 6 months of IRS spray & 131 & 17.8 \\
\hline Others preventions methods & 38 & 4.96 \\
\hline - Others preventions methods = smoking in the house, take tablets and using Aerosol spray \\
\hline
\end{tabular}


In the FGD discussion, the IRS operation has a long history in their locality about half of the respondents explained that their house was sprayed in the last 12 months. The majority of the discussants participated in the IRS by preparing their house and fetching water for spray preparation. A 50 years old female participant in Abaytera kebele explained "we prepare the house for spray by renewing the wall, take out of materials which we use in the house, we keep away home animals and children for some hours, and fetch the water for spray."

As the discussant expressed there were few refusals and plastering of the wall in six month of spray due to different reasons such as miss-understanding of IRS chemicals; miss-conception of the chemical as a multiplier of other insect nuisance; and the current IRS chemical is less efficacious and expires in a short time to kill mosquitoes than the previous brands (i.e. DDT). A 60 years old male in Menti kebele FGD explained "The current yellow color IRS chemical is less efficacious and expires in a short time."

In the discussions, the majority of the participants did not own LLINs. All groups in the discussions indicated that LLINs' inaccessibility to buy and lack of provision appeared to contribute to the low household insecticide bed net ownership. A 39 years old male in Abaytera kebele FGD explained "for the last time the bed net was distributed three years ago." Somewhat surprisingly, in the discussion, some of them mentioned that there was the use of LLINs for others beyond the intended purpose. In Kalay kebele FGD participants, "We use the bed net for other purposes due to old age of the LLINs and lack of

awareness." Some of the discussants also mentioned that nowadays there is a decrement in the efficacy of LLINs brands.

The discussants participated in environmental management (compact, drain, and clearing the vegetation) in Abaytera FGD "we participate in malaria prevention in environmental management using 1 to 5 group male and female groups and the group leaders organize every activity."

\section{Determinants of practice towards malaria prevention and control}

From the multivariable model, it was found that those individuals who live in Menti kebele had about 4 times increased odds of having a good practice as compared with respondents who live in Aswagari kebele $[\mathrm{AOR}=3.88,95 \% \mathrm{Cl}: 2.43,6.20]$. The odds of having good practice is decreased by $35 \%$ among female respondents as compared to male respondents [AOR $=0.65,95 \% \mathrm{Cl}: 0.47,0.90$,]. The odds of having a good practice is decreased by $66 \%$ among illiterate respondents as compared to respondents who had formal education [AOR $=0.34,95 \% \mathrm{Cl}: 0.16,0.72$. Moreover, the odds of having a good practice is decreased by $55 \%$ among respondents in the poorest wealth quintile as compared to the wealthiest [AOR $=0.45,95 \% \mathrm{Cl}: 0.27,0.76]$. Similarly the odds of having good practice was decreased by $49 \%, 76 \%$, and $79 \%$ among respondents in poor, Medium and wealthy wealth quintiles respectively as compared with wealthiest wealth quintiles with $[\mathrm{AOR}=0.51,95 \% \mathrm{Cl}: 0.30,0.88]$; $[\mathrm{AOR}=0.24 ; 95 \% \mathrm{Cl}: 0.14,0.42]$; and $[A O R=0.2195 \% \mathrm{Cl}: 0.12,0.36]$ respectively. The odds of having good practice was decreased by $48 \%$ 
among respondents who have poor knowledge about malaria prevention as compared to their counterparts [AOR $=0.52,95 \% \mathrm{Cl}: 0.36,0.75]$. $($ Table 3$)$

Table 3

determinants of malaria prevention and control Practice in West Belessa, North West Ethiopia, 2019.

\begin{tabular}{|c|c|c|c|c|c|c|}
\hline \multirow[t]{2}{*}{ Variable } & \multirow[t]{2}{*}{ Response } & \multicolumn{5}{|c|}{ Level of Practice } \\
\hline & & Good (\%) & Poor (\%) & $\operatorname{COR}(95 \% \mathrm{Cl})$ & AOR(95\%Cl) & $\begin{array}{l}\mathrm{P} \text { - } \\
\text { value }\end{array}$ \\
\hline \multirow[t]{5}{*}{$\begin{array}{l}\text { Resident's } \\
\text { Kebele }\end{array}$} & Kalay & $39(5.3)$ & $70(9.5)$ & $\begin{array}{l}0.68(0.42- \\
1.11)\end{array}$ & $\begin{array}{l}0.62(0.37- \\
1.05)\end{array}$ & 0.08 \\
\hline & Abaytera & $56(7.6)$ & $94(12.7)$ & $\begin{array}{l}0.73(0.47- \\
1.14)\end{array}$ & $\begin{array}{l}0.68(0.43- \\
1.09)\end{array}$ & 0.11 \\
\hline & Menti & $160(21.7)$ & $48(6.5)$ & $\begin{array}{l}4.10(2.66- \\
6.32)\end{array}$ & $\begin{array}{l}3.88(2.43- \\
6.20)\end{array}$ & $\begin{array}{l}< \\
0.001\end{array}$ \\
\hline & Amstya & $38(5.1)$ & $48(6.5)$ & $\begin{array}{l}0.97(0.58- \\
1.63)\end{array}$ & $\begin{array}{l}0.99(0.57- \\
1.72)\end{array}$ & 0.96 \\
\hline & Aswagari & $83(11.2)$ & 102(13.8) & 1 & 1 & \\
\hline \multirow[t]{2}{*}{ Sex } & Female & 139(18.8) & $189(25.6)$ & $\begin{array}{l}0.54(0.4- \\
0.72)\end{array}$ & $\begin{array}{l}0.65(0.47- \\
0.90)\end{array}$ & 0.01 \\
\hline & Male & $237(32.1)$ & $173(23.3)$ & 1 & 1 & \\
\hline \multirow[t]{3}{*}{$\begin{array}{l}\text { Educational } \\
\text { status }\end{array}$} & Illiterate & $317(43.0)$ & $331(44.9)$ & $\begin{array}{l}0.38(0.19- \\
0.76)\end{array}$ & $\begin{array}{l}0.34(0.16- \\
0.72)\end{array}$ & 0.005 \\
\hline & Literate & $29(3.9)$ & $19(2.6)$ & $\begin{array}{l}0.27(0.61- \\
1.486)\end{array}$ & $\begin{array}{l}0.39(0.15- \\
1.04)\end{array}$ & 0.06 \\
\hline & $\begin{array}{l}\text { Formal } \\
\text { education }\end{array}$ & $30(4.1)$ & $12(1.6)$ & 1 & 1 & \\
\hline \multirow[t]{5}{*}{ Wealth index } & Poorest & $82(11.1)$ & $85(11.5)$ & $\begin{array}{l}0.37(0.23- \\
0.60)\end{array}$ & $\begin{array}{l}0.45(0.27- \\
0.76)\end{array}$ & 0.003 \\
\hline & Poor & 77(10.4) & $62(8.4)$ & $\begin{array}{l}0.48(0.29- \\
0.79)\end{array}$ & $\begin{array}{l}0.51(0.30- \\
0.88)\end{array}$ & 0.02 \\
\hline & Medium & $86(11.7)$ & $56(7.6)$ & $\begin{array}{l}0.25(0.15- \\
0.41)\end{array}$ & $\begin{array}{l}0.24(0.14- \\
0.42)\end{array}$ & $\begin{array}{l}<.001 \\
0.001\end{array}$ \\
\hline & Wealthy & $90(12.2)$ & $60(8.1)$ & $\begin{array}{l}0.26(0.16- \\
0.42)\end{array}$ & $\begin{array}{l}0.21(0.12- \\
0.36)\end{array}$ & $\begin{array}{l}<.001 \\
0.001\end{array}$ \\
\hline & Wealthiest & 101(13.7) & $39(5.3)$ & 1 & 1 & \\
\hline \multirow[t]{2}{*}{$\begin{array}{l}\text { Knowledge } \\
\text { level }\end{array}$} & Poor & $61(8.3)$ & $116(15.7)$ & $\begin{array}{l}0.41(0.29- \\
0.58)\end{array}$ & $\begin{array}{l}0.52(0.36- \\
0.75\end{array}$ & 0.001 \\
\hline & Good & $315(42.7)$ & $245(33.2)$ & 1 & 1 & \\
\hline
\end{tabular}




\section{Discussion}

This study mainly investigated malaria prevention and control practices and associated factors among rural households in malarious areas of West Belessa.

The major malaria targets of the Ministry of Health are a $100 \%$ household coverage of LLINs in the family household in all malarious areas with the free distribution of LLINs.(8) Since 2005 significantly increased the coverage of the LLINs intervention. $(15,16)$ despite this in west Belessa only $21.3 \%$ of the respondents owned at least one LLINs in their house. This is much lower as compared with studies done in Woreta and Arba Minch as well as the 2015 MIS $(64 \%)(8,17,18)$ The difference is due to lack of access to buy the bed net and lack of affordability as confirmed in FGD discussion. This evidence is supported by a study done in southern Tanzania.(19) Regarding utilization of the total respondents who owned at least one LLINs $68.6 \%$ of them used it in the previous night of data collection this is in line with a study done in Gurage and a study done in Dabat district Amhara region. $(10,20)$

The FMOH's National Malaria Strategic Plan aims to provide $100 \%$ IRS coverage as a key malaria prevention measure in areas where the malaria burden is high. According to this survey, $80.5 \%$ of the houses were sprayed with IRS in the past 12 Months this is higher compared with the MIS 2015 which was $29 \%$, and a study done in Gondar town. $(8,21)$ But it is lower compared with a study done in Jabi tehnan Ethiopia (87.5\%).(22) The difference may be due to the difference in the study area. Because only 14.85 of the country's total population targeted for IRS spray.(23) So that areas targeted for IRS have high coverage of IRS. In this study, $84.3 \%$ of the respondents participated in environmental management (i.e. compact, drainage, and Clear vegetation) to control malaria. This is similar to a study done in the Gurage zone and in Eritrea. $(10,16)$ In this study, the respondents report that there were low prevention practices of other malaria prevention methods i.e. aerosol spray, and take a tablet which was used by about only $5 \%$ of the respondents in total. Similarly, only $3.7 \%$ of the respondents used closing window or doors at an early time to prevent malaria. This is lower comparing with the studies in Pawe and Shewa robit.(24, 25) This is due to low knowledge of these prevention methods as confirmed in the FGD which was mentioned by none of the participants. This evidence is also supported by a study done in West Belessa as well as in Nyando Kenya. $(11,26)$

Overall, $50.9 \%$ of the study participants had good practice in comprehensive with regard to the practice of malaria prevention and control. This is in line with the study done in Amhara National Regional State, Ethiopia, in which $47.1 \%$ of the total study participants have good practice towards malaria prevention and control activities.(27) However, it is lower than a study done in Areka (67.7\%).(13) This could be due to study area differences our study has been conducted in rural areas which are difficult to get malaria prevention options such as LLINs and others while other studies conducted in urban setups.

In this survey, the odds of having a good practice is $35 \%$ less among female participants as compared with male participants. This is consistent with a study done in Nigeria.(12) This could be due to that male individuals are responsible for malaria prevention activities especially in rural households prevention activities outside the house are responsibilities for males. 
The odds of having a good practice is $66 \%$ less among illiterate respondents as compared with formally educated educational status. As information is the first step for practice households lead by illiterate individuals were less likely to practice malaria prevention practice than formally educated individuals; results from some other researches also reported a higher probability of malaria prevention measures undertaken by educated individuals. $(28,29)$ The odds of having good practice were $55 \%, 49 \%, 76 \%$, and $79 \%$ less among poorest, poor, Medium, and wealthy wealth quintiles respectively as compared with wealthiest wealth quintiles. Households with the lowest economic status were less likely to practice prevention measures than the higher economic status households. Similar to this finding, higher practice amongst households with a higher wealth index has been reported previously in Gurage(10). This could be due to the fact that individuals from high wealth index households have better information on access and capacity to buy sample-monetary aerosol sprays, build a favorable house for living and hence are more likely to use bed nets, spray houses and drain compound. The odds of having good practice are 3 times increased among respondents who live in Menti kebele as compared with Aswagari kebele this was related to good malaria prevention knowledge, as evidenced by a study done in West Belessa.(26)

\section{Conclusion}

In west Belessa; there were good IRS and environmental management malaria prevention practices, however, LLINs and other malaria prevention methods such as using the aerosol spray, screening windows and doors as well as closing windows and doors at an early time were poorly practiced. There was a lack of accessibility and provision of LLINs in the study area. As confirmed through FGD nowadays there is a decrement in the efficacy of IRS and LLINs as compared with the previous brands. Wealth index, malaria prevention knowledge, respondents living kebele, educational level, and respondents' sex were predictors for malaria prevention practice.

The use of easily accessible personal protection methods such as screening windows and doors; closing windows and doors early at night as well as other malaria prevention methods such as using aerosol spray are recommended malaria prevention methods. Programmers, partners, and implementers are recommended to increase the accessibility of LLINs in different options such as free provision and easily accessible at the market. Further research is needed for the efficacy of the IRS and LLIN. Low socioeconomic groups, low educational status, and female individuals need attention in malaria prevention and control programs.

\section{Abbreviations}

AOR: Adjusted Odds Ratio; Cl: Confidence Interval; FGD: Focus Group Discussions; FMOH: Federal Ministry of Health; IQR: Inter Quartile Range; IRS: Indoor Residual Spray; LLINs: Long Lasting Insecticidal Nets; OR: Odds Ratio; SD: Standard Deviation; UNICEF: United Nations Children's Fund; WHO: World Health Organization; DDT: Dichloro-diphenyl-trichloroethane

\section{Declarations}




\section{Ethical approval and consent to participate}

Approval to carry out the study was sought and obtained from the University Of Gondar Ethics Review Board. Written consent was obtained from all study participants after a detailed explanation of the purpose of the study.

\section{Availability of data and materials}

The data could be accessed based on request

\section{Funding}

Not applicable

\section{Consent for publication}

Not applicable

\section{Competing Interests}

There is no competing interest.

\section{Authors' contribution}

Tsigie B. was Conception and design, acquisition of data, or analysis and interpretation of data. Haile ab F. drafting the article or revising contents. All authors read and approved the final version of the manuscript.

\section{Acknowledgments}

We would like to thank West Belessa District administrative bodies and community cooperation and permission to conduct the study. We are also thankful for the University of Gondar for their support to conduct the study as well as the data collectors who participated in the study for their commitment. I would like to thank president's malaria initiative (PMI) for their financial support.

\section{References}

1. World Health Organisation. Global technical Strategy for Malaria 2016-2030. 2015; 
2. Organization WH. World malaria report 2020: 20 years of global progress and challenges. 2020;

3. Ethiopian federal ministry of health,. National Strategic plan for malaria prevention, control and elimination in Ethiopia, 2010 - 2015, (2002/2003 -2007/2008 EC Addis Ababa,. 2009 Mar;

4. Aregawi M, Lynch M, Bekele W, Kebede H, Jima D, Taffese HS, et al. Time series analysis of trends in malaria cases and deaths at hospitals and the effect of antimalarial interventions, 2001-2011, Ethiopia. PLoS One. 2014;9(11):e106359.

5. US-president's malaria initiative Ethiopia, Malaria-operational-plan. 2017.

6. Organization WH. Regional Office for Africa "Implementation of Indoor Residual Spraying of Insecticides for Malaria Control." November; 2007.

7. US-president's malaria initiative Ethiopia. Malaria operational-plan. 2018;

8. Ethiopian Public Health Institute. Malaria-Indicator-Survey. Vol. 6. 2015.

9. Dako-Gyeke M, Kofie HM. Factors influencing prevention and control of malaria among pregnant women resident in urban slums, Southern Ghana. Afr J Reprod Health. 2015;19(1):44-53.

10. Girum T, Hailemikael G, Wondimu A. Factors affecting prevention and control of malaria among endemic areas of Gurage zone: an implication for malaria elimination in South Ethiopia, 2017. Trop Dis Travel Med Vaccines. 2017;3(1):17.

11. Mbijiwe KK. Factors Influencing Use of Malaria Control Methods among the Residents of Nyando Sub-County, Kisumu County. JKUAT-COHES; 2019.

12. Amusan VO, Umar YA, Vantsawa PA. Knowledge, attitudes and practices on malaria prevention and control among private security guards within Kaduna Metropolis, Kaduna State-Nigeria. Sci J Public Health. 2017;5:240-5.

13. Kebede DL, Hibstu DT, Birhanu BE, Bekele FB. Knowledge, Attitude and Practice towards Malaria and Associated Factors in Areka Town, Southern Ethiopia: Community-Based Cross Sectional Study. J Trop Dis. 2017;5(3):1-11.

14. World Health Organization. Guidelines for core population coverage indicators for Roll Back Malaria: to be obtained from household surveys. 2004.

15. Ethiopian ministry of health FDE. National malaria strategic plan 2014-2020. Addis Ababa Minist Health. 2014;

16. Habtai H, Ghebremeskel T, Mihreteab S, Mufunda J, Ghebremichael A. Knowledge, attitudes and practices (KAP) about malaria among people visiting referral hospitals of Eritrea in 2008. J Eritrean Med Assoc. 2009;4(1):42-6.

17. Alelign A, Petros B. Knowledge, attitudes and practices of malaria transmission and preventive measures in Woreta town, Northwest Ethiopia. BMC Public Health. 2018;11:491.

18. Astatkie A. Knowledge and practice of malaria prevention methods among residents of Arba Minch Town and Arba Minch Zuria District, Southern Ethiopia. Ethiop J Health Sci. 2010;20(3).

19. Nathan R, Masanja H, Mshinda H, Schellenberg JA, De Savigny D, Lengeler C, et al. Mosquito nets and the poor: can social marketing redress inequities in access? Trop Med Int Health. 
2004;9(10):1121-1126.

20. Muchie KF, Alemu K, Tariku A, Tsegaye AT, Abebe SM, Yitayal M, et al. Rural households at risk of malaria did not own sufficient insecticide treated nets at Dabat HDSS site: evidence from a cross sectional re-census. BMC Public Health. 2017;17(1):888.

21. Tilaye T. Assessment of malaria prevalence and knowledge attitude an d practices (KAP) in relation to malaria prevention and control in Gondar town. North Ethiop Cent Data Cat Addis Ababa Univ. 2016;

22. Animut A, Negash $Y$, Kebede N. Distribution and utilization of vector control strategies in a malarious village of Jabi Tehnan District, north-western Ethiopia. Malar J. 2014;13(1):356.

23. Initiative UPM. Ethiopia Malaria Operational Plan FY 2019. 2019.

24. Beyene HB, Telele NF, Mekuria AH. Knowledge, attitude and practice on malaria and associated factors among residents in Pawe district, north west Ethiopia: a cross-sectional study. Sci J Public Health. 2015;3(3):303-9.

25. Abate A, Degarege A, Erko B. Community knowledge, attitude and practice about malaria in a low endemic setting of Shewa Robit Town, northeastern Ethiopia. BMC Public Health. 2013;13(1):312.

26. Aragie TB. Knowledge of malaria prevention and control methods and associated factors among rural households in west Belessa district, north west Ethiopia, 2019. BMC Public Health. 2020 Aug 24;20(1):1275.

27. Aderaw Z, Gedefaw M. Knowledge, attitude and practice of the community towards malaria prevention and control options in anti-malaria association intervention zones of Amahara National Regional State, Ethiopia. J Trop Dis. 2013;

28. Girum T, Hailemikael G, Wondimu A. Factors affecting prevention and control of malaria among endemic areas of Gurage zone: an implication for malaria elimination in South Ethiopia, 2017. Trop Dis Travel Med Vaccines. 2017;3(1):17.

29. Luyiga F. Knowledge, attitude and practices on malaria prevention and control in Uganda. Publ Commun Dev Found Uganda CDFU Retrieved Online Httpwww Cdc GovMalariaMalariaworldwideindex Html. 2013;

\section{Figures}




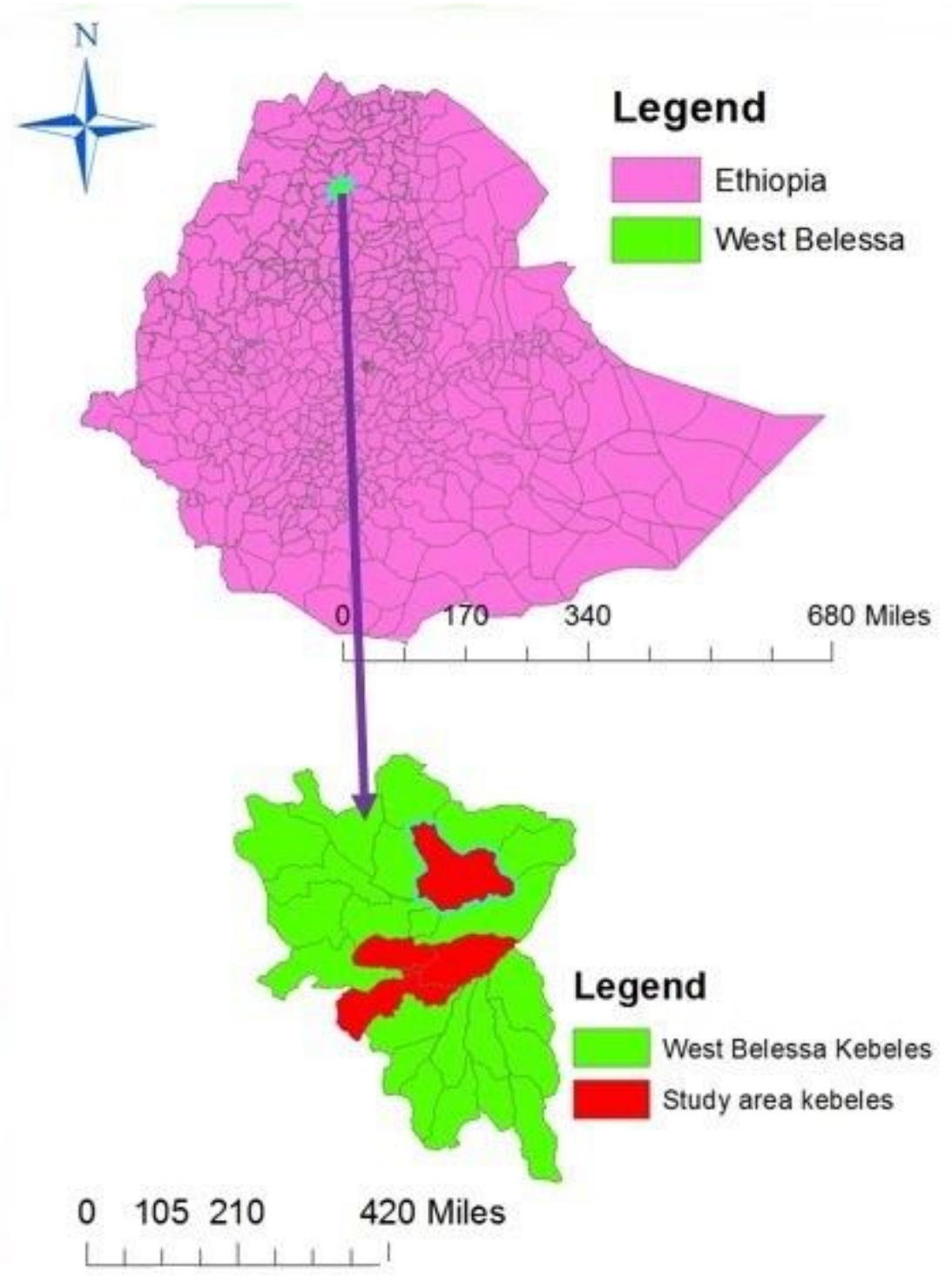

Figure 1

Location of study area, West Belessa, North west Ethiopia, 2019. Note: The designations employed and the presentation of the material on this map do not imply the expression of any opinion whatsoever on the part of Research Square concerning the legal status of any country, territory, city or area or of its authorities, or concerning the delimitation of its frontiers or boundaries. This map has been provided by the authors. 